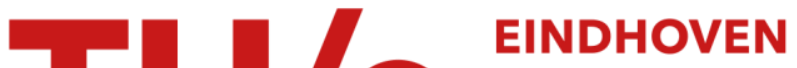 UNIVERSITY OF TECHNOLOGY
}

\section{Asymptotic behaviour of Toeplitz matrix in multi-input multi- output model predictive control}

\section{Citation for published version (APA):}

Tran, N. Q., Ozkan, L., Ludlage, J. H. A., \& Backx, A. C. P. M. (2013). Asymptotic behaviour of Toeplitz matrix in multi-input multi-output model predictive control. In 2013 European Control Conference (ECC), July 17-19, 2013, Zürich, Switzerland. (pp. 3784-3789). Institute of Electrical and Electronics Engineers.

Document status and date:

Published: $17 / 07 / 2013$

\section{Please check the document version of this publication:}

- A submitted manuscript is the version of the article upon submission and before peer-review. There can be important differences between the submitted version and the official published version of record. People interested in the research are advised to contact the author for the final version of the publication, or visit the $\mathrm{DOI}$ to the publisher's website.

- The final author version and the galley proof are versions of the publication after peer review.

- The final published version features the final layout of the paper including the volume, issue and page numbers.

Link to publication

\section{General rights}

Copyright and moral rights for the publications made accessible in the public portal are retained by the authors and/or other copyright owners and it is a condition of accessing publications that users recognise and abide by the legal requirements associated with these rights.

- Users may download and print one copy of any publication from the public portal for the purpose of private study or research.

- You may not further distribute the material or use it for any profit-making activity or commercial gain

- You may freely distribute the URL identifying the publication in the public portal.

If the publication is distributed under the terms of Article 25fa of the Dutch Copyright Act, indicated by the "Taverne" license above, please follow below link for the End User Agreement:

www.tue.nl/taverne

Take down policy

If you believe that this document breaches copyright please contact us at:

openaccess@tue.nl

providing details and we will investigate your claim. 


\title{
Asymptotic behaviour of Toeplitz matrix in multi-input multi-output model predictive control
}

\author{
Quang N. Tran*, Leyla Özkan*, Jobert Ludlage ${ }^{\dagger}$ and A.C.P.M. Backx* \\ ${ }^{*}$ Department of Electrical Engineering \\ Eindhoven University of Technology, Eindhoven, The Netherlands \\ Email: N.Q.Tran@tue.nl, L.Ozkan@tue.nl and A.C.P.M.Backx@tue.nl \\ ${ }^{\dagger}$ Delft Center for Systems and Control \\ Delft, The Netherlands \\ Email: J.H.A.Ludlage@tudelft.nl
}

\begin{abstract}
The singular value decomposition of (SVD) of the Toeplitz matrix in the quadratic performance index of Model Predictive Control (MPC) is studied. The underlying goal is to find connection between the frequency domain information and the finite time optimal control and use this connection as a basis for stability, robust performance analysis and tuning of the dynamic MPC criterion. In a recent work by the authors, it was shown that the singular value decomposition of the Toeplitz matrix provides gain and phase information of the associated system for sufficiently long prediction and control horizons. This work is extended to MIMO case and is shown that singular value decomposition of the Toeplitz matrix can be used for stability analysis of closed loop system.
\end{abstract}

\section{INTRODUCTION}

In refining and petrochemical industries, model predictive control (MPC) is the standard control technology due to its ability to make the system operate closely to its operational constraints ([1]). At each time instant, MPC solves an optimisation problem based on a model of the plant. The solution to the optimisation problem is the future sequence of control inputs and only the first element of the sequence is implemented on the plant. When the next measurement is available, the same procedure is repeated.

A major problem of any model-based operation system is that the lifetime performance degrades over time if proper supervision is not performed. Besides the changes in the disturbances, the changes in dynamics of the real plant due to material ageing or changes in operating condition may also result in a poor performance. A good maintenance strategy for model-based systems is then needed for retaining a good lifetime performance in the presence of such model uncertainty.

Control design that deals with uncertainty is investigated in depth using frequency domain approaches ([2], [3], [4]). Moreover, frequency-domain information is directly connected to the natural behaviour of the system and shows how close to instability the system is. To use similar frequencydomain-based techniques in designing finite-time MPC systems, a relation between the two domains is needed. To this end, initial studies were done in [5], [6], and [7]. They showed the link between the singular values of the Hessian matrix and the gain of the associated system in the frequency domain. In [8], the singular value decomposition technique is used to analyse the asymptotic behaviour of the Toeplitz matrix in single-input-single-output (SISO) systems. In [9] and [10], a similar technique was used to investigate the characteristics of inner systems and non-minimum phase behaviour.

This paper builds on those results and studies the behaviour of the Toeplitz matrix for multivariable systems. Section II gives background on MPC and also introduces it in the framework of an internal model control (IMC) scheme. Section III discusses the asymptotic behaviour of the Toeplitz matrix and Section IV provides results on the link to closed-loop stability. Section V illustrates the results with an example and Section VI discusses potential future research directions.

\section{PRELIMINARIES}

MPC computes the future behaviour of the system based on a prediction model. Assume a system of $n_{y}$ outputs and $n_{u}$ inputs is described by the impulse response model:

$$
y(k)=\sum_{i=0}^{\infty} H(i) u(k-i)
$$

where $H(i) \in \mathbb{R}^{n_{y} \times n_{u}}$ is the $i^{t h}$ element of the impulse response sequence. The sequence of predicted output of a multivariable system of $n_{y}$ outputs and $n_{u}$ inputs is calculated as follows:

$$
Y_{f}=H_{a} U_{p}+T U_{f}
$$

where $U_{p} \in \mathbb{R}^{M n_{u} \times 1}, U_{f} \in \mathbb{R}^{N n_{u} \times 1}$ and $Y_{f} \in \mathbb{R}^{P n_{y} \times 1}$ denote the vector of past inputs up to the past horizon $M$, the vector of the future inputs up to the control horizon $N$ and the future outputs up to the prediction horizon $P$, respectively: 


$$
\begin{aligned}
Y_{f}= & {\left[\begin{array}{c}
y(k) \\
y(k+1) \\
\vdots \\
y(k+P-2) \\
y(k+P-1)
\end{array}\right] ; U_{p}=\left[\begin{array}{c}
u(k-M) \\
\vdots \\
u(k-2) \\
u(k-1)
\end{array}\right] } \\
U_{f}= & {\left[\begin{array}{c}
u(k) \\
u(k+1) \\
\vdots \\
u(k+N-2) \\
u(k+N-1)
\end{array}\right] }
\end{aligned}
$$

$T \in \mathbb{R}^{P n_{y} \times N n_{u}}$ is the Toeplitz matrix:

$T=\left(\begin{array}{ccccc}H(0) & 0 & \ldots & \ldots & 0 \\ H(1) & H(0) & 0 & \ldots & 0 \\ H(2) & H(1) & H(0) & \ddots & \vdots \\ \vdots & \vdots & \vdots & \ddots & 0 \\ H(N-1) & H(N-2) & H(N-3) & \ldots & H(0) \\ H(N) & H(N-1) & H(N-2) & \ldots & H(1) \\ \vdots & \vdots & \vdots & & \vdots \\ H(P-1) & H(P-2) & H(P-3) & \ldots & H(P-N)\end{array}\right)$

and $H_{a} \in \mathbb{R}^{M n_{u} \times P n_{y}}$ is the Hankel matrix:

$$
H_{a}=\left(\begin{array}{cccc}
H(M) & H(M-1) & \ldots & H(1) \\
H(M+1) & H(M) & \ldots & H(2) \\
\vdots & \vdots & & \vdots \\
H(P+M-1) & H(P+M-2) & \ldots & H(P)
\end{array}\right)
$$

At each iteration, the MPC solves the minimisation problem of the quadratic cost function:

$$
\min _{U_{f}} V=\left\|Y_{\text {ref }}-Y_{f}\right\|_{Q}^{2}+\left\|U_{f}\right\|_{R}^{2}
$$

where $Q$ and $R$ are the weighting matrices on output errors and inputs respectively, $Y_{\text {ref }} \in \mathbb{R}^{P n_{y}}$ is the reference trajectory. From (2), it implies that:

$$
V=\left\|Y_{\text {ref }}-T U_{f}-H_{a} U_{p}\right\|_{Q}^{2}+\left\|U_{f}\right\|_{R}^{2}
$$

The solution to optimisation problem (5) is given by:

$$
\begin{aligned}
U_{f} & =\left(T^{\top} Q T+R\right)^{-1} T^{\top} Q\left(Y_{\text {ref }}-H_{a} U_{p}\right) \\
& =\mathcal{H}^{-1} T^{\top} Q\left(Y_{\text {ref }}-H_{a} U_{p}\right)
\end{aligned}
$$

where $\mathcal{H}$ is the Hessian matrix. When $Q=I, R=0, N=P$ and $T$ is of full rank, the solution $U_{f}$ reduces to:

$$
U_{f}=T^{-1}\left(Y_{\text {ref }}-H_{a} U_{p}\right)
$$

The first element of $U_{f}$ is implemented until the next measurement is available. In the case of no active constraints, this control can be considered as an approximate inverse of

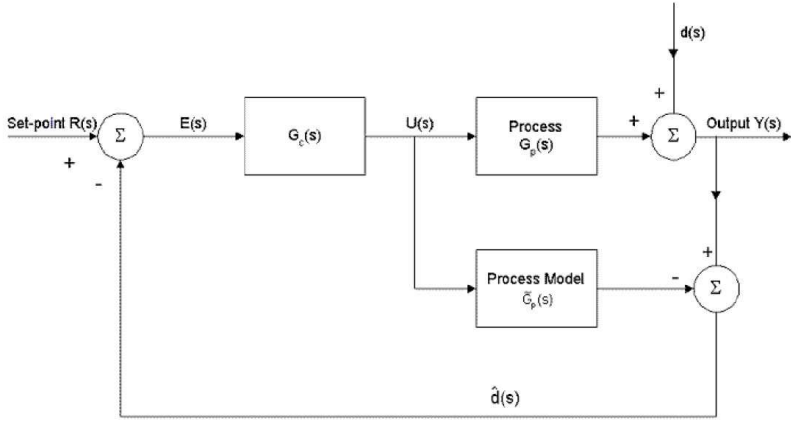

Fig. 1. IMC scheme

the process transfer matrix. When the prediction horizon is infinite, it is equivalent to an IMC scheme given in Fig. 1. The equivalence between MPC and IMC is elaborated in [11].

From the IMC scheme in Fig. 1, it follows that:

$$
\begin{aligned}
& Y=d+\left(I+G_{c}\left(G_{p}-\tilde{G}_{p}\right)\right)^{-1} G_{p} G_{c}(R-d) \\
& U=\left(I+G_{c}\left(G_{p}-\tilde{G}_{p}\right)\right)^{-1} G_{c}(R-d)
\end{aligned}
$$

Hence, the stability of the closed-loop system depends on the term $\left(I+G_{c}\left(G_{p}-\tilde{G}_{p}\right)\right)^{-1}$.

Assume the plant has input uncertainty:

$$
G_{p}=\tilde{G}_{p}(I+\Delta)
$$

it implies that

$$
\left(I+G_{c}\left(G_{p}-\tilde{G}_{p}\right)\right)^{-1}=\left(I+G_{c} \tilde{G}_{p} \Delta\right)^{-1}
$$

Therefore, the open-loop characteristics of $G_{c} \tilde{G}_{p}$ are investigated to analyse the the stability of the closed-loop system, based on Nyquist-like techniques ([12] and [3]). In order to apply these techniques to the finite-time domain MPC, the behaviour of the Toeplitz matrix is studied.

\section{ASYMPTOTIC BEHAVIOUR OF THE TOEPLitZ MATRIX FOR THE MIMO CASE}

The behaviour of the Toeplitz matrix in the SISO case is presented in [8]. It is shown that the magnitude and phase of the frequency response of a SISO system can be found in the singular values and singular vectors of the Toeplitz matrix. The singular values of the Toeplitz matrix are the gain of the open-loop system and the singular vectors give corresponding phase information. For the MIMO case, the SVD of the Toeplitz matrix is also studied. It is shown in [6] that the singular values of the Toeplitz matrix are exactly the singular values of the frequency response when the frequency varies from 0 to $\pi$. Here, we investigate the phase and directionality information in the singular values and how this information is linked to the stability of the closed-loop system. 


\section{A. Gain, phase and directionality information in the Toeplitz} matrix

For the sake of simplicity, we only consider square systems $\left(n_{u}=n_{y}\right)$. Consider a square $n_{i o} \times n_{i o}$ open-loop system and its frequency response $G\left(e^{j \omega}\right)$. The singular value decomposition of $G\left(e^{j \omega}\right)$ is given by

$$
G\left(e^{j \omega}\right)=\mathcal{U}\left(e^{j \omega}\right) \Sigma(\omega) \mathcal{V}^{*}\left(e^{j \omega}\right)
$$

where $\mathcal{U} \in \mathbb{C}^{n_{i o} \times n_{i o}}, \mathcal{V} \in \mathbb{C}^{n_{i o} \times n_{i o}}$ and $\Sigma(\omega)=$ $\operatorname{diag}\left\{\sigma_{1}(\omega), \ldots, \sigma_{n_{i o}}(\omega)\right\}$. It can be deduced from [6] that in the MIMO case, the eigenvalues of the Hessian matrix converge to the singular values of the frequency response (i.e. $\sigma_{i}(\omega)$ ), with the following matrix of eigenvectors:

$$
E_{N, \omega}=\frac{1}{\sqrt{N}}\left[\begin{array}{c}
\mathcal{V}\left(e^{j \omega}\right) \\
e^{j \omega} \mathcal{V}\left(e^{j \omega}\right) \\
\vdots \\
e^{j(N-1) \omega} \mathcal{V}\left(e^{j \omega}\right)
\end{array}\right]
$$

with $\omega=\frac{\pi}{N} p, p \in 0, \ldots, N-1$. Let $\mathcal{V}\left(e^{j \omega}\right)=$ $\left[\begin{array}{llll}\mathfrak{v}_{1} & \mathfrak{v}_{2} & \cdots & \mathfrak{v}_{n_{i o}}\end{array}\right]$, it follows that in a certain input direction $\mathfrak{v}_{i}$ (of size $1 \times n_{i o}$ ), the following holds in the limit $N \rightarrow \infty$ :

$$
\mathcal{H}\left[\begin{array}{c}
\mathfrak{v}_{i} \\
e^{j \omega} \mathfrak{v}_{i} \\
\vdots \\
e^{j(N-1) \omega_{\mathfrak{v}_{i}}}
\end{array}\right]=\sigma_{i}^{2}(\omega)\left[\begin{array}{c}
\mathfrak{v}_{i} \\
e^{j \omega} \mathfrak{v}_{i} \\
\vdots \\
e^{j(N-1) \omega_{\mathfrak{v}_{i}}}
\end{array}\right]
$$

where $\mathcal{H}$ is the Hessian matrix and $\sigma_{i}(\omega)$ is $i^{t h}$ element of $\Sigma(\omega)$ (associated with direction $i$ ). Since the Hessian matrix is the square of the Toeplitz matrix, it implies that $\sigma_{i}(\omega)$ are the singular values of the Toeplitz matrix and the corresponding right singular vector is (note that index $i$ indicates the direction and $p$ indicates the frequency in the range $[0 ; \pi])$ :

$$
V_{i, p}=\frac{1}{\sqrt{N}}\left[\begin{array}{c}
\mathfrak{v}_{i} \\
e^{j \omega} \mathfrak{v}_{i} \\
\vdots \\
e^{j(N-1) \omega_{\mathfrak{v}_{i}}}
\end{array}\right]
$$

Since the vector above is complex and the Hessian and Toeplitz matrices are real, we introduce their real singular vectors as follows. Let

$$
\mathfrak{v}_{i}=\left[\begin{array}{c}
A_{i 1} e^{j \varphi_{i 1}} \\
A_{i 2} e^{j \varphi_{i 2}} \\
\vdots \\
A_{i 2 n_{i o}} e^{j \varphi_{i n_{i o}}}
\end{array}\right]
$$

Note that $\omega=\frac{\pi}{N} p$ with $p \in\{0, \ldots, N-1\}$, which makes $\omega$ vary from 0 to $\pi$. As the singular values $\sigma_{i}(\omega)$ are real, the real right singular vectors of the Toeplitz matrix are just the real part of the complex singular vectors and given by:

$$
V_{i, p}=\sqrt{\frac{2}{N}}\left[\begin{array}{c}
A_{i 1} \cos \left(\varphi_{i 1}\right) \\
A_{i 2} \cos \left(\varphi_{i 2}\right) \\
\vdots \\
A_{i n_{i o}} \cos \left(\varphi_{i n_{i o}}\right) \\
A_{i 1} \cos \left(\varphi_{i 1}+\omega\right) \\
A_{i 2} \cos \left(\varphi_{i 2}+\omega\right) \\
\vdots \\
A_{i n_{i o}} \cos \left(\varphi_{i n_{i o}}+\omega\right) \\
\vdots \\
\vdots \\
A_{i 1} \cos \left(\varphi_{i 1}+(N-1) \omega\right) \\
A_{i 2} \cos \left(\varphi_{i 2}+(N-1) \omega\right) \\
\vdots \\
A_{i n_{i o}} \cos \left(\varphi_{i n_{i o}}+(N-1) \omega\right)
\end{array}\right]
$$

The singular vectors $V_{i, p}$ now have two indices: $i$ is the input direction associated with $V_{G i}\left(e^{j \omega}\right)$ in the frequency domain and $p$ is the index which shows its frequency content. When $p$ varies from 0 to $N-1$ and $i$ from 1 to $n_{i o}$, expression (19) generates $N . n_{i o}$ right singular vectors, which is consistent with the size $N n_{i o} \times N n_{i o}$ of the Toeplitz matrix. We now propose the following theorem that relates the phase and directional information of the system with the singular vectors of the Toeplitz matrix

Theorem 1: Consider a square system of size $n_{i o} \times n_{i o}$ and its frequency response $G\left(e^{j \omega}\right)$. Its SVD is given by

$$
G\left(e^{j \omega}\right)=\mathcal{U}\left(e^{j \omega}\right) \Sigma(\omega) \mathcal{V}^{*}\left(e^{j \omega}\right)
$$

with $\mathcal{V}\left(e^{j \omega}\right)=\left[\begin{array}{llll}\mathfrak{v}_{1} & \mathfrak{v}_{2} & \cdots & \mathfrak{v}_{n_{i o}}\end{array}\right]$ and $\mathcal{U}\left(e^{j \omega}\right)=$ $\left[\begin{array}{llll}\mathfrak{u}_{1} & \mathfrak{u}_{2} & \cdots & \mathfrak{u}_{n_{\text {io }}}\end{array}\right]$. As shown in [13], the angle between singular subspaces (i.e. directional information) is defined as

$$
\phi(i, \omega)=\arccos \left\|\mathfrak{v}_{i}^{*}\left(e^{j \omega}\right) \mathfrak{u}_{i}\left(e^{j \omega}\right)\right\|
$$

and the phase difference between singular vectors is given by

$$
\Delta \theta(i, \omega)=\arg \left(\mathfrak{v}_{i}^{*}\left(e^{j \omega}\right) \mathfrak{u}_{i}\left(e^{j \omega}\right)\right)
$$

Consider the Toeplitz matrix $T \in \mathbb{R}^{N n_{i o} \times N n_{\text {io }}}$ with prediction horizon $N$

$$
T=\left(\begin{array}{cccccc}
H(0) & 0 & \ldots & \ldots & \ldots & 0 \\
\vdots & \ddots & \ddots & & & \vdots \\
H\left(k_{0}\right) & \ldots & H(0) & 0 & & \vdots \\
0 & \ddots & & \ddots & \ddots & \vdots \\
\vdots & \ddots & \ddots & & \ddots & 0 \\
0 & \ldots & 0 & H\left(k_{0}\right) & \ldots & H(0)
\end{array}\right)
$$


where $H(i)=\left[\begin{array}{cccc}h_{11}(i) & h_{12}(i) & \cdots & h_{1 n_{i o}}(i) \\ h_{21}(i) & h_{22}(i) & \cdots & h_{2 m}(i) \\ \vdots & \vdots & \ddots & \vdots \\ h_{n_{i o} 1} & h_{n_{i o} 2} & \cdots & h_{n_{i o} n_{i o}}(i)\end{array}\right]$ is the matrix of impulse response of each pair of input-output at time instant $i$. The SVD of $T$ is given by: $T=U S V^{\top}$, with

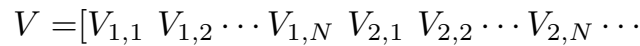

$$
\begin{aligned}
& \left.\cdots V_{n_{i o}, 1} V_{n_{i o}, 2} \cdots V_{n_{i o}, N}\right]
\end{aligned}
$$

where $V_{i, p}$ is given in (19) and similarly

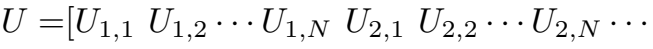

$$
\begin{aligned}
& \left.\cdots U_{n_{i o}, 1} U_{n_{i o}, 2} \cdots U_{n_{i o}, N}\right]
\end{aligned}
$$

$S$ is a $N n_{i o} \times N n_{i o}$ diagonal matrix of the principal gains of the frequency response. Then, in the limit $N \rightarrow \infty$, the following equality holds:

$$
U_{i, p}^{\top} V_{i, p}=\cos (\phi(i, \omega)) \cos (\Delta \theta(i, \omega))
$$

with $\omega=\frac{p}{N} \pi, p \in\{0, \ldots, N-1\}, i \in\left\{1, \ldots, n_{i o}\right\}$.

Proof:

From the relation between the gain of the system and the singular values of the Toeplitz matrix described above and (16), it follows that

$$
\begin{aligned}
& T V_{i, p}=\sigma_{i}(\omega) U_{i, p} \\
\Rightarrow & V_{i, p}^{\top} T V_{i, p}=\sigma_{i}(\omega) V_{i, p}^{\top} U_{i, p}
\end{aligned}
$$

where $T$ is given in 23 and $V_{i, p}$ in 19 . Using the trigonometric identity:

$$
\cos x \cos y=\frac{1}{2}(\cos (x+y) \cos (x-y)) \text { for } x ; y \in \mathbb{R}
$$

after some calculations, we obtain

$$
\begin{aligned}
& V_{i, p}^{\top} T V_{i, p}=\operatorname{Re}\left\{\frac{1}{N}\left[\begin{array}{cccc}
\mathfrak{v}_{i}^{*} & e^{-j \omega_{\mathfrak{v}_{i}^{*}}^{*}} & \cdots & e^{-j(N-1) \omega_{\mathfrak{v}_{i}^{*}}}
\end{array}\right]\right. \\
& \left.\left(\begin{array}{cccccc}
H(0) & 0 & \cdots & \cdots & \cdots & 0 \\
\vdots & \ddots & \ddots & & & \vdots \\
H\left(k_{0}\right) & \ldots & H(0) & 0 & & \vdots \\
0 & \ddots & & \ddots & \ddots & \vdots \\
\vdots & \ddots & \ddots & & \ddots & 0 \\
0 & \ldots & 0 & H\left(k_{0}\right) & \cdots & H(0)
\end{array}\right)\left[\begin{array}{c}
e^{j \mathfrak{v}_{i}} \\
\vdots \\
e^{j(N-1) \omega_{\mathfrak{v}_{i}}}
\end{array}\right]\right\} \quad(30) \\
& =\operatorname{Re}\left\{\frac { 1 } { N } \left(N v_{i}^{*} H(0) \mathfrak{v}_{i}+(N-1) \mathfrak{v}_{i}^{*} e^{-j \omega} H(1) \mathfrak{v}_{i}+\cdots\right.\right. \\
& \left.\left.+\left(N-k_{0}\right) \mathfrak{v}_{i}^{*} e^{-j \omega k_{0}} H\left(k_{0}\right) \mathfrak{v}_{i}\right)\right\} \\
& =\operatorname{Re}\left\{\mathfrak{v}_{i}^{*} H(0) \mathfrak{v}_{i}+\frac{N-1}{N} v_{i}^{*} e^{-j \omega} H(1) \mathfrak{v}_{i}+\cdots\right. \\
& \left.+\frac{N-k_{0}}{N} \mathfrak{v}_{i}^{*} e^{-j \omega k_{0}} H\left(k_{0}\right) \mathfrak{v}_{i}\right\}
\end{aligned}
$$

In the limit $N \rightarrow \infty, \frac{N-k}{N} \rightarrow 0$ with $k$ constant. Hence, the equality above leads to

$$
\begin{aligned}
V_{i, p}^{\top} T V_{i, p} & =\operatorname{Re}\left\{\mathfrak{v}_{i}^{*}\left(\sum_{l=0}^{k_{0}} e^{-j \omega l} H(l)\right) \mathfrak{v}_{i}\right\} \\
& =\operatorname{Re}\left\{\mathfrak{v}_{i}^{*} G\left(e^{j \omega}\right) \mathfrak{v}_{i}\right\} \\
\Rightarrow \operatorname{Re}\left(V_{i, p}^{\top} T V_{i, p}\right) & =\operatorname{Re}\left(\mathfrak{v}_{i}^{*} G\left(e^{j \omega}\right) \mathfrak{v}_{i}\right)
\end{aligned}
$$

On the other hand, we have

$$
\begin{aligned}
G\left(e^{j \omega}\right) \mathfrak{v}_{G i} & =\sigma_{i}(\omega) \mathfrak{u}_{G i} \\
\Rightarrow \mathfrak{v}_{G i}^{*} G\left(e^{j \omega}\right) \mathfrak{v}_{G i} & =\sigma_{i}(\omega) \mathfrak{v}_{G i}^{*} \mathfrak{u}_{G i} \\
& =\sigma_{i} \cos \phi(i, \omega) e^{j \Delta \theta(i, \omega)} \\
\Rightarrow \operatorname{Re}\left(\mathfrak{v}_{G i}^{*} G\left(e^{j \omega}\right) \mathfrak{v}_{G i}\right) & =\sigma_{i} \cos (\phi(i, \omega)) \cos (\Delta \theta(i, \omega))
\end{aligned}
$$

From (28), (34) and (39), it follows that

$$
U_{i, p}^{\top} V_{i, p}=\cos (\phi(i, \omega)) \cos (\Delta \theta(i, \omega))
$$

\section{(Q.E.D)}

\section{LINK TO THE STABILITY OF THE CLOSED-LOOP SYSTEM}

From Theorem 1, it is shown (in equality (26)) that the inner product of the left and right singular vectors of the Toeplitz matrix in the time domain gives the multiplication of the directional angle and phase angle of the corresponding left and right singular vectors of the frequency response in the frequency domain. This section discusses the relation between this product and the stability of the closed-loop system with a unity feedback and provides preliminary results.

Consider the same system with frequency response $G\left(e^{j \omega}\right)$ as in the previous section. The eigendecomposition of $G\left(e^{j \omega}\right)$ is given by:

$$
G\left(e^{j \omega}\right)=\mathcal{W}^{*}\left(e^{j \omega}\right) \Lambda(\omega) \mathcal{W}^{*}\left(e^{j \omega}\right)^{-1}
$$

where $\Lambda(\omega)^{n_{i o} \times n_{i o}}$ is the diagonal matrix whose nonzero elements $\lambda_{i}(\omega)$ are the eigenvalues of $G\left(e^{j \omega}\right)$ and $\mathcal{W}\left(e^{j \omega}\right)^{n_{i o} \times n_{\text {io }}}$ is the matrix of the corresponding eigenvectors:

$$
\mathcal{W}^{*}\left(e^{j \omega}\right)=\left[\begin{array}{llll}
\mathfrak{w}_{1} & \mathfrak{w}_{2} & \ldots \mathfrak{w}_{n_{\text {io }}}
\end{array}\right]
$$

It is known that the stability of the closed-loop system with a unity feedback is determined based on the locus of the eigenvalues $\lambda_{i}(\omega)$ over the frequency range $[0 ; \pi]$. For a stable open-loop system, the closed-loop system is stable if the characteristic loci of the eigenvalues do not encircle the point $(-1 ; 0)$ ([12]). The closed-loop system is marginally stable if the locus cross the point $(-1 ; 0)$, i.e. there is some frequency where the eigenvalue is -1 . Let $\lambda_{i}(\omega)$ be an arbitrary eigenvalue associated with its eigenvector $\mathfrak{w}_{i}(\omega)$. The singular values and the pair of singular vectors 
of the frequency response at the corresponding frequency are denoted $\sigma_{i}(\omega)$ and $\mathfrak{u}_{i}(\omega), \mathfrak{v}_{i}(\omega)$, respectively.

In this paper, symmetric open-loop transfer matrices are considered. When the product of the controller and the process is symmetric, the directionality of the process is not affected by the controller. In a simple $2 \times 2$ system, it means that the weight on output 1 is equal to that on output 2 and the weight on input 1 is equal to that on input 2. It is shown in [9] that if the process is ill-conditioned, this choice is a decent tuning strategy to avoid aggressiveness of the inputs and to obtain more robustness for the system. For a symmetric open-loop system, the SVD of $G\left(e^{j \omega}\right)$ coincides with the eigen-decomposition, i.e. the eigenvalue matrix $\Lambda(\omega)$ are also the singular value matrix $\Sigma(\omega)$ and the eigenvectors $\mathfrak{w}_{i}$ are equal to the singular vectors $\mathfrak{v}_{i}$. Moreover, the SVD of the Toeplitz matrix gives the same information as the SVD of the frequency response as shown in previous sections. Hence, the characteristic loci of the eigenvalues of $G\left(e^{j \omega}\right)$ can also be found in SVD of the Toeplitz matrix.

\section{EXAMPLE}

Consider the following symmetric open-loop transfer matrix, which is supposed to be the product of the controller and the process model in the Laplace domain:

$$
\left[\begin{array}{cc}
\frac{1}{s^{2}+2 s+1} & \frac{1}{s+2} \\
\frac{1}{s+2} & \frac{1}{s+1}
\end{array}\right]
$$

The system is discretised with a sampling period of 1 minute. The characteristic loci of $\Lambda(\omega)$ is given in Fig. 2. The loci show that for each direction, the gain margins are $1 / 0.6941=1.4407$ and $1 / 0.2892=3.4578$, respectively. The characteristic loci of the system can be interpreted as the Nyquist plot of the multi-variable system. From the loci, one can derive the stability of the closed-loop system. In this example, the gain margins 1.4407 and 3.4578 show that if the gain of the open-loop system is increased by 1.4407 times without affecting the directionality, the closed-loop system will go unstable.

The Toeplitz matrix of the open-loop system is then constructed in order to show that the same information in the frequency domain can also be obtained from this time-domain-based matrix. The matrix is constructed with a horizon of 200 samples. The horizon is chosen to be high for illustrative reason, as the complete matching between the frequency-domain information and time-domain information is obtained when the horizon tends to infinity (Theorem 1). The SVD of the Toeplitz matrix is analysed to illustrate the results of Theorem 1. Fig. 3 illustrates equality (40), which shows that the inner product of the left and right singular vectors of the Toeplitz matrix coincide with that of the left and right singular vectors of the frequency response over the frequency range $[0, \pi]$. The frequency where the inner product is -1 is also the critical point of the characteristic loci. Fig. 4 shows that the singular values of the Toeplitz matrix match the singular values of the frequency response of the open-loop system. At the critical frequency where the inner
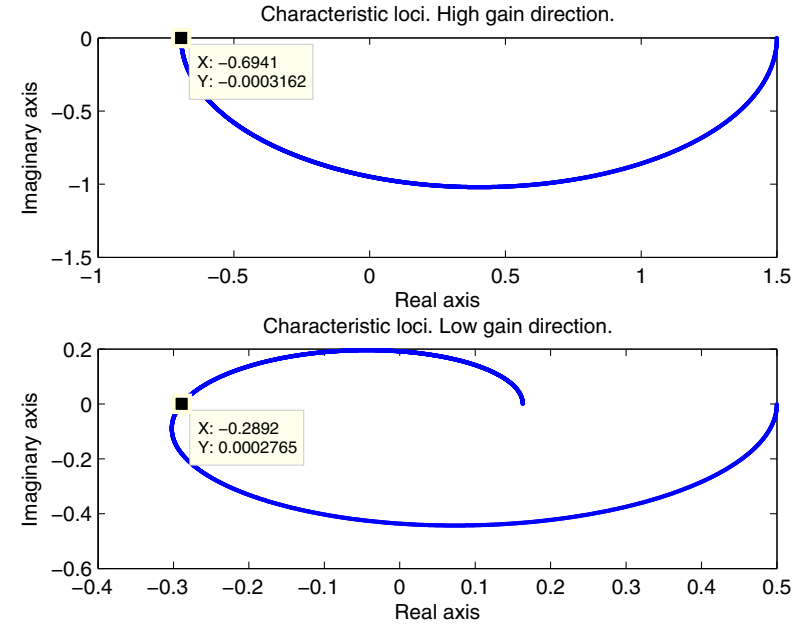

Fig. 2. Characteristic loci of the eigenvalues of the frequency response
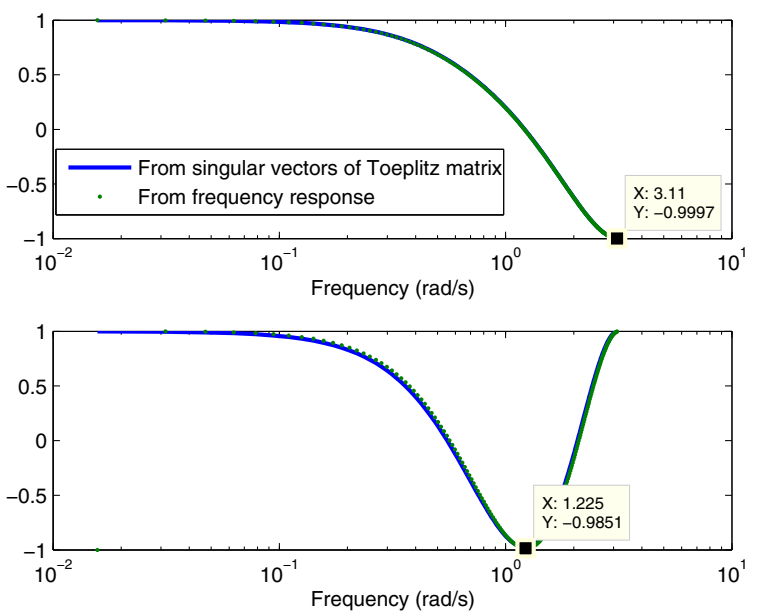

Fig. 3. Inner product of left and right singular vectors of the Toeplitz matrix coincides with the directional and phase information in the frequency domain

product of the singular vectors of the Toeplitz matrix is -1 , the same gain margins are obtained from the Toeplitz matrix for both directions $(-3.17 \mathrm{~dB}$ and $-10.98 \mathrm{~dB}$, respectively). In summary, the information on the stability of the closedloop system can be obtained from the SVD of the Toeplitz matrix. Note that the horizon is also the number of frequency points of the singular vectors taken from the Toeplitz matrix. Therefore, reducing the horizon will provide fewer frequency points, while the general matching of the two domains still remains.

\section{CONCLUSION AND FUTURE WORK}

The asymptotic behaviour of the Toeplitz matrix in symmetric multivariable systems is investigated. For such systems, the SVD of the frequency response coincides with the eigen-decomposition and the Toeplitz matrix can then cover all the frequency-domain characteristics of the systems. This can be considered as the first step to analyse the time- 

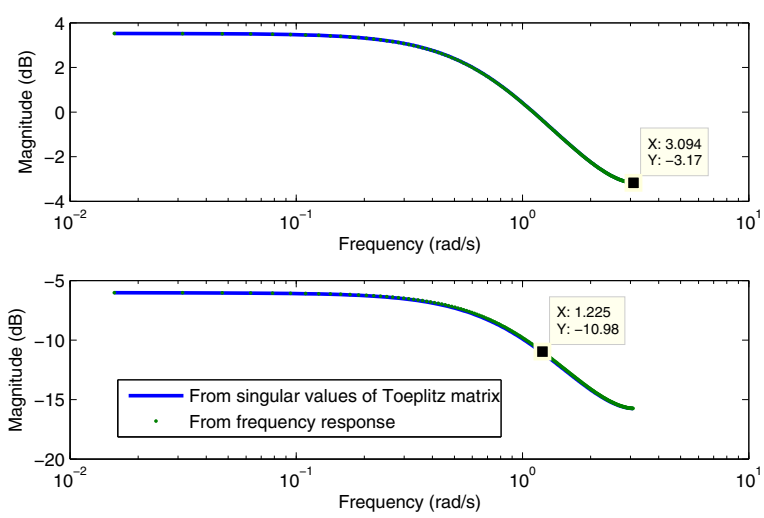

Fig. 4. Gain of the open-loop system. Gain margins coincide with the characteristic loci.

domain MPC based on frequency-domain techniques such as Nyquist-like techniques. To extend the work to the nonsymmetric case, the structure of the product of the singular vectors in different directions must be studied. Since most of the results are based on infinite horizon, an investigation into the effect of receding horizon and of the time-varying control is still needed. Then a proper choice of the horizons in MPC may follow.

\section{ACKNOWLEDGMENT}

The research leading to these results has received funding from the European Union's Seventh Framework Programme (FP7/2007-2013) under grant agreement $\mathrm{n}^{\circ} 257059$, the 'Autoprofit' project (www.fp7-autoprofit.eu).

\section{REFERENCES}

[1] S. J. Qin and T. A. Badgwell, "A survey of industrial model predictive control technology," Control Engineering Practice, vol. 11, pp. 733 764, 2003.

[2] M. Morari and E. Zafiriou, Robust Process Control. Englewood Cliffs, NJ: Prentice-Hall, Inc, 1989.

[3] K. Zhou, J. C. Doyle, and K. Glover, Robust and Optimal Control. New Jersey 07458: Prentice Hall, 1996.

[4] S. Skogestad and I. Postlethwaite, Multivariable feedback control: Analysis and Design. John Wiley and Sons Ltd, 2005.

[5] O. J. Rojas, G. C. Goodwin, A. Feuer, and M. M. Serón, "A sub-optimal receding horizon control strategy for constrained linear systems," Proceedings of the 2003 American Control Conference, June 2003.

[6] O. J. Rojas and G. C. Goodwin, "On the asymptotic properties of the hessian in discrete-time linear quadratic control," Proceedings of the 2004 American Control Conference, June-July 2004.

[7] O. J. Rojas, G. C. Goodwin, M. M. Serón, and A. Feuer, "An svd based strategy for receding horizon control of input constrained linear systems," International Journal of Robust and Nonlinear Control, vol. 14, pp. 1207-1226, 2004.

[8] Q. N. Tran, L. Özkan, J. Ludlage, and A. C. P. M. Backx, "Asymptotic characteristics of toeplitz matrix in siso model predictive control," IFAC Symposium on Advanced Control of Chemical Processes, 2012.

[9] J. H. Ludlage, "Controllability analysis of industrial processes: towards the industrial application,” Ph.D. dissertation, Eindhoven University of Technology, The Netherlands, 1997.

[10] J. H. Ludlage, S. Weiland, A. A. Stoorvogel, and A. C. P. M. Backx, "Finite-time behaviour of inner systems," IEEE Transactions on Automatic Control, vol. 48, no. 7, pp. 1134-1149, July 2003.

[11] C. E. Garcia and M. Morari, "Internal model control. a unifying review and some new results," Ind. Eng. Chem. Process Des. Dev., vol. 21, no. 2, pp. 308-323, 1982.
[12] J. M. Maciejowski, Multivariable Feedback Design. Addison-Wesley Publishers Ltd., 1989.

[13] J.S.Freudenberg and D.P.Looze, Frequency Domain Properties of Scalar and Multivariable Feedback Systems, M. Thoma and A.Wyner, Eds. Springer-Verlag, 1988. 